\title{
Microscopic origin of the mobility enhancement at a spinel/perovskite oxide heterointerface revealed by photoemission spectroscopy
}

Schuetz, P.; Christensen, Dennis Valbjørn; Borisov, V.; Pfaff, F.; Scheiderer, P.; Dudy, L.; Zapf, M.; Gabel, J.; Chen, Y. Z.; Pryds, Nini

Total number of authors:

18

Published in:

Physical Review B

Link to article, DOI:

10.1103/PhysRevB.96.161409

Publication date:

2017

Document Version

Publisher's PDF, also known as Version of record

Link back to DTU Orbit

Citation (APA):

Schuetz, P., Christensen, D. V., Borisov, V., Pfaff, F., Scheiderer, P., Dudy, L., Zapf, M., Gabel, J., Chen, Y. Z., Pryds, N., Rogalev, V. A., Strocov, V. N., Schlueter, C., Lee, T. -L., Jeschke, H. O., Valenti, R., Sing, M., \& Claessen, R. (2017). Microscopic origin of the mobility enhancement at a spinel/perovskite oxide heterointerface revealed by photoemission spectroscopy. Physical Review B, 96(16), [161409].

https://doi.org/10.1103/PhysRevB.96.161409

\section{General rights}

Copyright and moral rights for the publications made accessible in the public portal are retained by the authors and/or other copyright owners and it is a condition of accessing publications that users recognise and abide by the legal requirements associated with these rights.

- Users may download and print one copy of any publication from the public portal for the purpose of private study or research.

- You may not further distribute the material or use it for any profit-making activity or commercial gain

- You may freely distribute the URL identifying the publication in the public portal 


\title{
Microscopic origin of the mobility enhancement at a spinel/perovskite oxide heterointerface revealed by photoemission spectroscopy
}

\author{
P. Schütz, ${ }^{1}$ D. V. Christensen, ${ }^{2}$ V. Borisov,${ }^{3}$ F. Pfaff, ${ }^{1}$ P. Scheiderer, ${ }^{1}$ L. Dudy,,${ }^{1}$ M. Zapf,${ }^{1}$ J. Gabel, ${ }^{1}$ Y. Z. Chen, ${ }^{2}$ N. Pryds,${ }^{2}$ \\ V. A. Rogalev,,${ }^{1,4}$ V. N. Strocov, ${ }^{4}$ C. Schlueter,${ }^{5}$ T.-L. Lee, ${ }^{5}$ H. O. Jeschke, ${ }^{3}$ R. Valentí, ${ }^{3}$ M. Sing, ${ }^{1}$ and R. Claessen ${ }^{1}$ \\ ${ }^{1}$ Physikalisches Institut and Röntgen Center for Complex Material Systems (RCCM), Universität Würzburg, Am Hubland, \\ D-97074 Würzburg, Germany \\ ${ }^{2}$ Department of Energy Conversion and Storage, Technical University of Denmark, DK-4000 Roskilde, Denmark \\ ${ }^{3}$ Institute of Theoretical Physics, Goethe University Frankfurt am Main, D-60438 Frankfurt am Main, Germany \\ ${ }^{4}$ Swiss Light Source, Paul Scherrer Institut, CH-5232 Villigen, Switzerland \\ ${ }^{5}$ Diamond Light Source, Harwell Sciene and Innovation Campus, Oxfordshire OX11 ODE, United Kingdom
}

(Received 5 April 2017; revised manuscript received 11 August 2017; published 27 October 2017)

\begin{abstract}
The spinel/perovskite heterointerface $\gamma-\mathrm{Al}_{2} \mathrm{O}_{3} / \mathrm{SrTiO}_{3}$ hosts a two-dimensional electron system (2DES) with electron mobilities exceeding those in its all-perovskite counterpart $\mathrm{LaAlO}_{3} / \mathrm{SrTiO}_{3}$ by more than an order of magnitude, despite the abundance of oxygen vacancies which act as electron donors as well as scattering sites. By means of resonant soft $\mathrm{x}$-ray photoemission spectroscopy and $a b$ initio calculations, we reveal the presence of a sharply localized type of oxygen vacancies at the very interface due to the local breaking of the perovskite symmetry. We explain the extraordinarily high mobilities by reduced scattering resulting from the preferential formation of interfacial oxygen vacancies and spatial separation of the resulting 2DES in deeper $\mathrm{SrTiO}_{3}$ layers. Our findings comply with transport studies and pave the way towards defect engineering at interfaces of oxides with different crystal structures.
\end{abstract}

DOI: 10.1103/PhysRevB.96.161409

The search for high-mobility two-dimensional electron systems (2DESs) at atomically engineered transition metal oxide heterointerfaces is an ongoing endeavor, since the strong electronic correlations in partially occupied $d$ orbitals promise an even richer physical behavior than found in conventional semiconductor heterostructures [1]. However, the charge carrier mobilities in the most prominent complex oxide 2DES, found at the perovskite-perovskite heterointerface between the band insulators $\mathrm{LaAlO}_{3}$ and $\mathrm{SrTiO}_{3}$, still fall short of those in semiconductors by several orders of magnitude [2]. The hitherto-highest mobility in $\mathrm{SrTiO}_{3}$-based structures $\left(140000 \mathrm{~cm}^{2} / \mathrm{V} \mathrm{s}\right.$ at $2 \mathrm{~K}$ ) is found at the spinel/perovskite heterointerface between $\gamma-\mathrm{Al}_{2} \mathrm{O}_{3}$ thin films and $\mathrm{SrTiO}_{3}[3,4]$, thus making it a promising candidate for applications in oxide electronics or fundamental studies of quantum transport.

The mechanism of 2DES formation in $\mathrm{SrTiO}_{3}$-based heterostructures has been under debate for many years. The existence of a critical film thickness for metallicity at the epitaxial $\mathrm{LaAlO}_{3} / \mathrm{SrTiO}_{3}$ heterointerface has been associated with the polar discontinuity at the interface and the concomitant buildup of an electrostatic potential gradient as a function of film thickness, which may be countered by a charge redistribution to the interface [3,5-8]. Additionally, substantial oxygen vacancy $\left(\mathrm{V}_{\mathrm{O}}\right)$ doping within the $\mathrm{SrTiO}_{3}$ substrate may occur, which is the dominant source of charge carriers in $2 \mathrm{DESs}$ discovered in $\mathrm{SrTiO}_{3}$ interfaced with amorphous overlayers $[9,10]$ and bare $\mathrm{SrTiO}_{3}$ surfaces irradiated with ultraviolet light [11-13]. In the case of $\gamma-\mathrm{Al}_{2} \mathrm{O}_{3} / \mathrm{SrTiO}_{3}$, it has been argued that its $2 \mathrm{DES}$ originates exclusively from oxygen vacancies in $\mathrm{SrTiO}_{3}$, which are formed due to redox reactions [3]. However, it remains unclear how high mobilities can be achieved, when $\mathrm{SrTiO}_{3}$ hosts both conduction electrons and oxygen vacancies, which act as scattering sites. Here, we reveal the existence of a specific type of oxygen vacancies at the spinel/perovskite $\gamma-\mathrm{Al}_{2} \mathrm{O}_{3} / \mathrm{SrTiO}_{3}$ interface by means of soft $\mathrm{X}$-ray resonant photoemission spectroscopy (SX-ResPES) and $a b$ initio calculations, and propose a spatial separation of the dopants (oxygen vacancies) and the 2DES as the origin for its exceptionally high mobility.

SX-ResPES experiments were performed at the combined soft and hard X-ray beamline I09, Diamond Light Source and at the soft x-ray beamline ADRESS, Swiss Light Source [14,15]. Theoretical calculations were performed based on the density functional theory in the generalized gradient approximation (GGA) [16-24] by explicitly including oxygen vacancies in a $\gamma-\mathrm{Al}_{2} \mathrm{O}_{3} / \mathrm{SrTiO}_{3}$ superlattice and a $\mathrm{SrTiO}_{3}$ supercell. For theoretical and experimental details including sample fabrication, see the Supplemental Material [25].

While the $\gamma-\mathrm{Al}_{2} \mathrm{O}_{3} / \mathrm{SrTiO}_{3}$ heterointerface can host a 2DES with extraordinarily high electron mobilities upon careful control of the deposition parameters [3], for a wide range of growth conditions, a lower-mobility 2DES forms [4]. We have found that such lower mobilities can be increased postgrowth by gentle low-temperature annealing or prolonged storage at room temperature, as shown in Fig. 1(a). The electron density of a $\gamma-\mathrm{Al}_{2} \mathrm{O}_{3} / \mathrm{SrTiO}_{3}$ sample obtained from standard Hall measurements in the van der Pauw geometry [3] remains largely unaffected in this process. In contrast, the mobility at $2 \mathrm{~K}$ and the residual resistance ratio $\left[R_{S}(300 \mathrm{~K}) / R_{S}(2 \mathrm{~K})\right]$ increase by roughly a factor of $4\left(12000-40000 \mathrm{~cm}^{2} / \mathrm{V} \mathrm{s}\right.$ and 730-3200, respectively).

Since the movement of larger cations is frozen at room temperature [26], cation diffusion can be excluded as the driving mechanism. In contrast, the diffusion of lighter oxygen anions (and hence oxygen vacancies) is feasible and may affect the transport properties at cryogenic temperatures, where electron scattering by ionized donors is the dominant effect [27]. Since the total amount of oxygen vacancies, as reflected in 
(a)

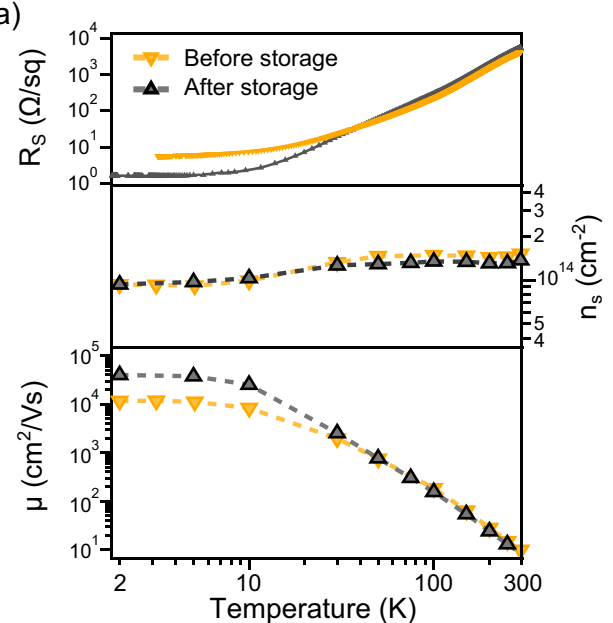

(b)

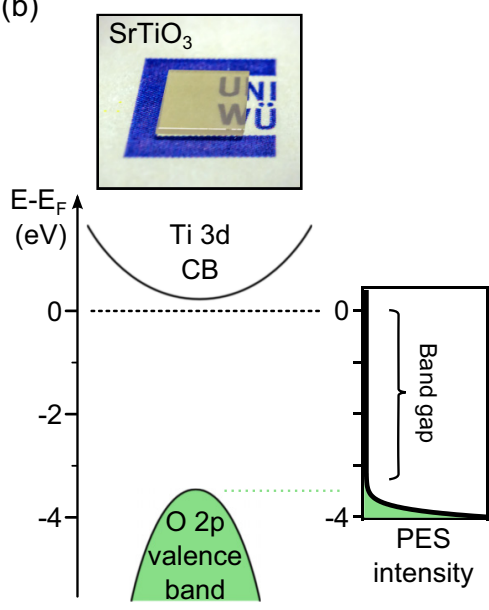

(c)

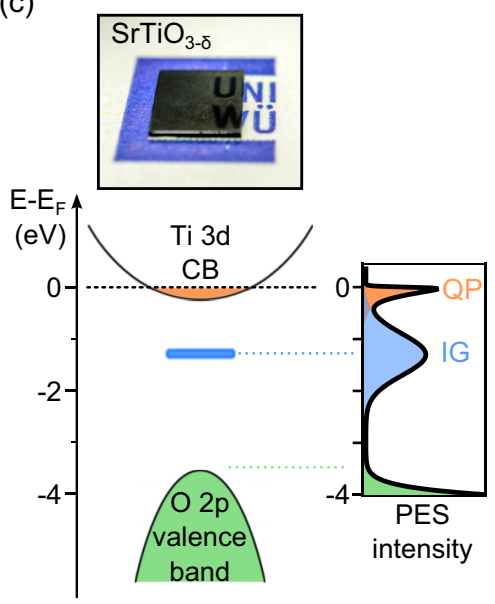

FIG. 1. (a) Sheet resistivity $\left(R_{S}\right)$, carrier density $\left(n_{S}\right)$, and mobility $(\mu)$ as a function of temperature $T$ of a $\gamma-\mathrm{Al}_{2} \mathrm{O}_{3} / \mathrm{SrTiO}_{3}$ heterostructure before and after 6 months of storage at room temperature in a vacuum desiccator. (b) Photograph, electronic structure, and corresponding schematic photoemission spectrum of stoichiometric $\mathrm{SrTiO}_{3}$ and (c) oxygen-deficient $\mathrm{SrTiO}_{3-\delta}$.

the charge carrier density, remains constant, one may speculate that a gradual redistribution of oxygen vacancy scattering sites away from the confined 2DES may be the cause for the mobility enhancement.

As shown in Fig. 1(b), nominally stoichiometric $\mathrm{SrTiO}_{3}$ is an intrinsically $n$-doped wide-gap semiconductor with the Fermi energy pinned close to the conduction band minimum [28]. Upon the introduction of oxygen vacancies, two electrons per vacancy become released and the electronic structure of $\mathrm{SrTiO}_{3}$ changes, as depicted in Fig. 1(c). A fraction of donor electrons becomes trapped in a localized Ti $3 d$-derived state next to the vacancy site (blue) and the rest is donated into the $\mathrm{Ti} 3 d$ conduction band (orange) to become itinerant $[29,30]$. The resulting photoemission spectrum comprises a dispersive feature cut off by the Fermi-Dirac distribution at the chemical potential, the quasiparticle peak (QP), and a broad nondispersive in-gap peak (IG), which can be seen as a hallmark for the presence of oxygen vacancies in $\mathrm{SrTiO}_{3}$ [31-33].

Here, we use synchrotron-based soft x-ray resonant photoemission spectroscopy (SX-ResPES) at the Ti $L$ edge to enhance the spectroscopic contrast for the Ti $3 d$-derived states at the buried interface [34,35]. The inset of Fig. 2(a) shows the PES spectra of $\mathrm{LaAlO}_{3} / \mathrm{SrTiO}_{3-\delta}$ and $\gamma-\mathrm{Al}_{2} \mathrm{O}_{3} / \mathrm{SrTiO}_{3-\delta}$ at the resonance condition. Despite an overall similarity, i.e., the presence of a QP and an IG peak, the latter is significantly broader and asymmetric for $\gamma-\mathrm{Al}_{2} \mathrm{O}_{3} / \mathrm{SrTiO}_{3-\delta}$, signaling a superposition of (at least) two peaks. Indeed, upon tuning the photon energy across the Ti $L$ absorption edge as shown in Fig. 2(a), two peaks at $E \approx-1.2 \mathrm{eV}$ (in-gap feature A, IGA) and $-2.1 \mathrm{eV}$ (IGB) can be distinguished by their shifted resonances $(h v=458.2$ and $459.1 \mathrm{eV}$ for IGB and IGA, respectively). Note that in these spectra the quasiparticle was suppressed by a specific choice of the measurement geometry [25].

It is well known that the exposure of maximal-valence transition metal oxides such as $\mathrm{SrTiO}_{3}$ to high-intensity synchrotron light causes oxygen vacancy formation [36]. This can be counteracted by simultaneous dosing with molecular oxygen, which dissociates in the high-intensity $\mathrm{x}$-ray beam and diffuses as atomic species into the substrate where it annihilates vacancies [13,37]. As shown in Fig. 2(b), using a metal capillary to direct oxygen onto the sample, IGB can be quenched entirely, whereas IGA is reduced, yet remains finite even for the highest possible oxygen flow. Thus, we conclude that both IG peaks represent oxygen vacancy sites, but apparently of different kinds. The different susceptibility to oxygen dosing indicates a different vertical spatial distribution of the two kinds of vacancies, i.e., the completely quenched IGB has its origin in closer proximity to the interface. Note that the shifted $\mathrm{O} 2 p$ valence band onset is due to variable band bending in $\mathrm{SrTiO}_{3}$ and a concomitant change of the band alignment at the interface.

By acquiring the photoemission signal under various emission angles $\theta$ with respect to the surface normal, the effective probing depth can be changed as $\lambda_{\text {eff }}=\lambda \cos \theta$ [38], where $\lambda$ is the inelastic mean free path. Figure 2(c) shows the resonant Ti $3 d$ spectral weight as a function of $\lambda_{\text {eff }}$, ranging from $1.2 \mathrm{~nm}$ (maximum bulk sensitivity, $\theta=0^{\circ}$ ) to $0.8 \mathrm{~nm}$ (maximum interface sensitivity, $\theta=45^{\circ}$ ) [39]. Additionally, the Ti $2 p$ core level signal excited by the second-order light from the undulator is seen above the chemical potential and serves as a bulk titanium reference signal. The relative spectral weights were extracted from Gaussian fits and are shown in the inset as a function of emission angle. The considerable angle dependence of the IGA-to-IGB-intensity ratio (red) provides evidence for an extraordinarily sharp, vertical separation of the two types of oxygen vacancies, i.e., IGB is situated closer to the interface. Furthermore, IGA scales with the second-order light-induced Ti $2 p$ bulk signal (blue), indicating a uniform distribution of IGA states throughout the $\mathrm{SrTiO}_{3}$ substrate (within the information depth of roughly $1.2 \mathrm{~nm}$ ). We thus readily identify IGA as a signal from bulklike oxygen vacancies situated within the substrate and IGB as a fingerprint of oxygen vacancies at the interface. The existence of two distinct types of oxygen vacancies-derived here from PES in a dynamical equilibrium situation inherently different from the near-thermodynamical state probed in transport experiments- 

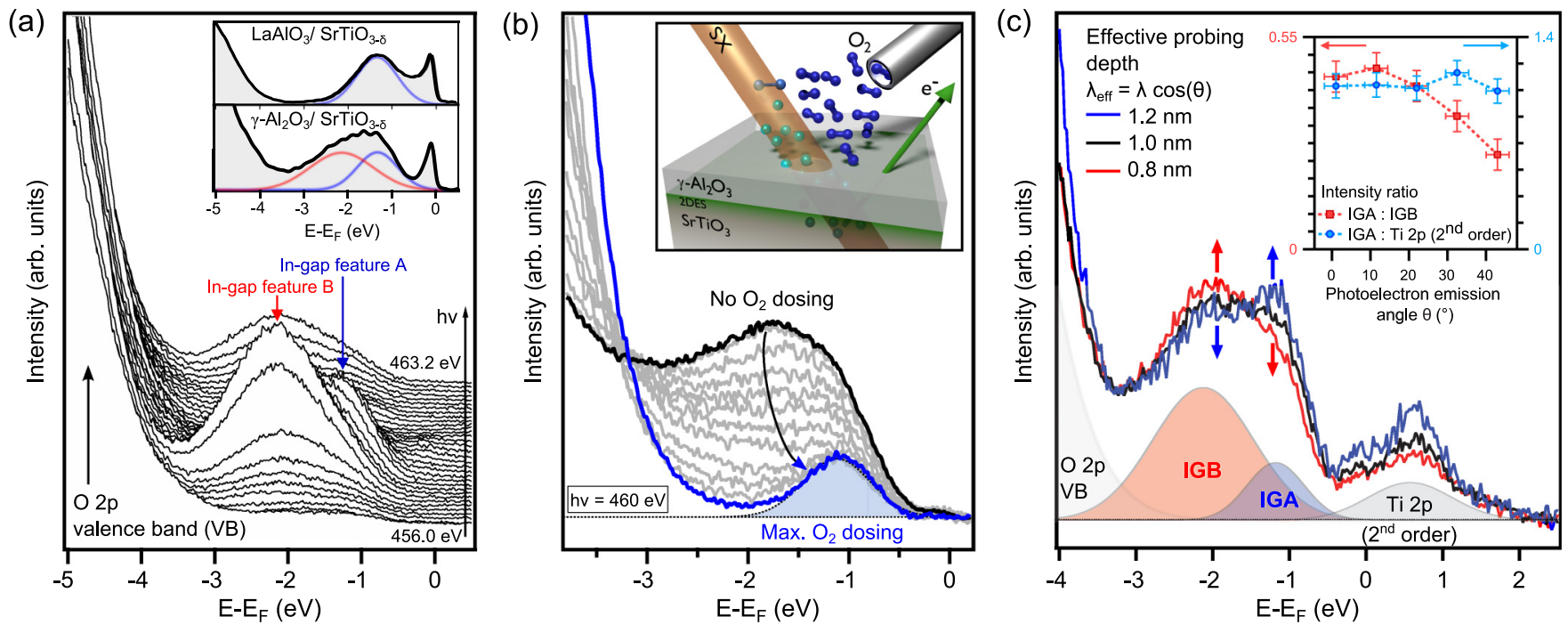

FIG. 2. (a) Photoemission spectra of a $\gamma-\mathrm{Al}_{2} \mathrm{O}_{3} / \mathrm{SrTiO}_{3}$ heterostructure upon tuning the photon energy across the Ti $L$ absorption edge Two in-gap features, IGA and IGB, are distinguished by their shifted resonance photon energies. Inset: Comparison between the systems $\mathrm{LaAlO}_{3} / \mathrm{SrTiO}_{3-\delta}$ and $\gamma-\mathrm{Al}_{2} \mathrm{O}_{3} / \mathrm{SrTiO}_{3-\delta}(h v=459.4 \mathrm{eV}$ ). (b) Resonant Ti $3 d$ spectral weight upon oxygen dosing (schematically depicted in the inset). While IGB becomes quenched entirely, a finite IGA weight remains at maximum dosing. (c) Depth-resolved photoemission spectroscopy of the Ti $3 d$ in-gap states and the second-order light-induced Ti $2 p$ core level $(h v=459.8 \mathrm{eV})$. The spectra are normalized to the spectral weight between $E=-0.5$ and $-3.5 \mathrm{eV}$. Inset: Relative spectral weight as a function of photoemission angle. The strong IGA-to-IGB angle dependence evidences the sharp vertical separation of IGA and IGB.

is nonetheless of high relevance also for the latter situation, as we sketch out in the following.

In a qualitative model, the introduction of a single oxygen vacancy into bulk $\mathrm{SrTiO}_{3}$ results in one itinerant electron in the Ti $3 d t_{2 g}$ band and one trapped electron in a bonding state derived from adjacent Ti $3 d e_{g} / 4 p_{z}$ hybrid states [29,30,40]. Indeed, as shown in Fig. 3(a), the Ti $3 d$-projected density of states (PDOS) obtained from $\mathrm{GGA}+U$ calculations of a $3 \times 3 \times 3 \mathrm{SrTiO}_{3}$ supercell with one oxygen vacancy, using the standard parameters $U=5 \mathrm{eV}$ and $J_{\mathrm{H}}=0.64 \mathrm{eV}$, exhibits metallic carriers and an IG state at $-1 \mathrm{eV}$, in good agreement with experiment and the qualitative model. The real-space charge density for a (100) layer cutting through the vacancy and obtained from energy integration of the in-gap state shows a typical Ti $3 d e_{g}$-based bonding orbital [29], which is strongly localized at the oxygen vacancy and its adjacent Ti atoms.

In $\mathrm{SrTiO}_{3}$-based all-perovskite heterostructures such as $\mathrm{LaAlO}_{3} / \mathrm{SrTiO}_{3}$, all oxygen vacancies in the substrate can be regarded as bulklike to a good approximation. In contrast, the perovskite symmetry is broken at the $\gamma-\mathrm{Al}_{2} \mathrm{O}_{3} / \mathrm{SrTiO}_{3}$ heterointerface since $\gamma-\mathrm{Al}_{2} \mathrm{O}_{3}$ adopts a spinel crystal structure [41-43]. As seen in Fig. 3(b), here, the local ionic coordination of an oxygen vacancy in the uppermost $\mathrm{TiO}_{2}$ layer (type B) differs significantly from that in deeper, bulklike layers (type A). The peculiar spinel cation sublattice lifts the local $C_{4 v}$ symmetry relevant for oxygen vacancies in bulk $\mathrm{SrTiO}_{3}$, likely resulting in states of different orbital compositions [44] and binding energies, as supported by a crystal-field analysis [25].

Figure 3(c) shows $\mathrm{GGA}+U$ calculations of a $\gamma-\mathrm{Al}_{2} \mathrm{O}_{3} / \mathrm{SrTiO}_{3}$ superlattice with one oxygen vacancy in the interfacial $\mathrm{TiO}_{2}$ layer. The $\mathrm{Ti} 3 d$-projected density of states exhibits two IG peaks (denoted as $\mathrm{IGB}_{1}$ and $\mathrm{IGB}_{2}$ ) at $E \approx-2.3$ and $-1.5 \mathrm{eV}$, that are hosted by the two adjacent inequivalent Ti cations. As expected, the real-space charge density maps corresponding to $\mathrm{IGB}_{1}$ and $\mathrm{IGB}_{2}$ show a rather complex $d$-orbital composition that is, however, reminiscent of $e_{g}$-like orbitals. The experimentally observed broad peak at $\approx-2.1 \mathrm{eV}$ (indicated by a guide-to-the-eye curve) results from oxygen vacancy clustering that will occur in any realistic system and leads to a statistical distribution of in-gap states with slightly different binding energies. Note that the formation enthalpy $\Delta H$ of single oxygen vacancies located in different $\mathrm{SrTiO}_{3}$ layers exhibits a minimum at the interface [Fig. 3(b)], hence suggesting a favored formation of type B oxygen vacancies and/or diffusion of vacancies from the bulk to the interface. A recent annealing study of $\gamma-\mathrm{Al}_{2} \mathrm{O}_{3} / \mathrm{SrTiO}_{3}$ indeed identified such diffusion processes, in support of the mechanism proposed here [45].

Figure 4(a) schematically summarizes our experimental and theoretical findings. The peculiar local symmetry breaking at the spinel/perovskite heterointerface $\gamma-\mathrm{Al}_{2} \mathrm{O}_{3} / \mathrm{SrTiO}_{3}$ results in a unique type of (interfacial) oxygen vacancy neither found in bulk $\mathrm{SrTiO}_{3}$ nor at its perovskite/perovskite counterpart $\mathrm{LaAlO}_{3} / \mathrm{SrTiO}_{3}$. In $\mathrm{SrTiO}_{3}$-based heterostructures oxygen vacancies represent an extrinsic source of electrons [46,47], but also act as strong scatterers for charge carriers when present near the 2DES. As already pointed out by Huijben et al., their deleterious effect on the mobility requires design strategies to remove them from the transport region, e.g., by a postgrowth exposure to an oxygen-rich environment at elevated temperature and possibly an incorporation of a $\mathrm{SrCuO}_{2}$ nanolayer that facilitates oxygen surface exchange $\left(\mu \approx 50000 \mathrm{~cm}^{2} / \mathrm{V} \mathrm{s}\right)$ [48]. However, in contrast to $\mathrm{LaAlO}_{3} / \mathrm{SrTiO}_{3}$, the $\gamma-\mathrm{Al}_{2} \mathrm{O}_{3} / \mathrm{SrTiO}_{3}$ heterointerface be- 
(a)

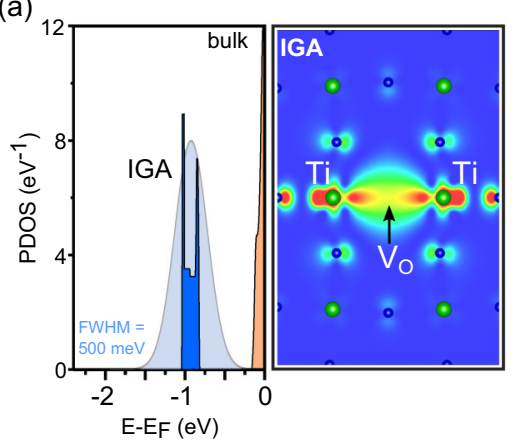

(b)

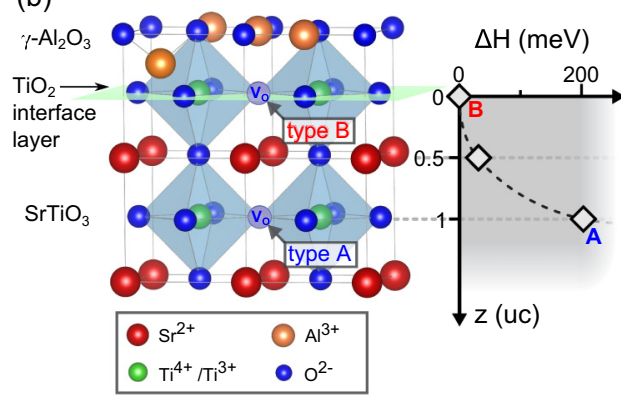

(c)

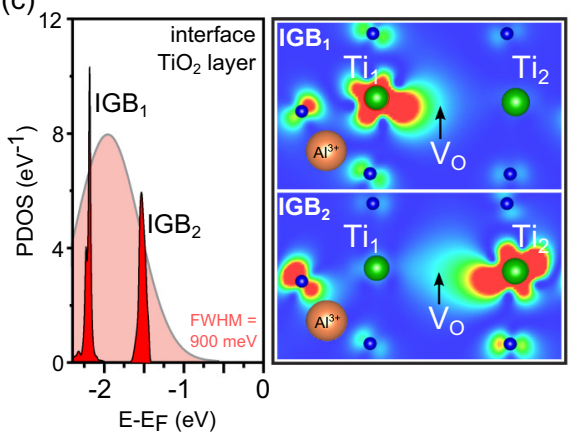

FIG. 3. (a) GGA $+U$ calculated Ti $3 d$-projected density of states (PDOS) and real-space electron density of bulk $\mathrm{SrTiO}_{3}$ with an isolated oxygen vacancy. The real-space charge map is shown for a (100) layer cutting the oxygen vacancy and obtained through energy integration of the IGA state. The experimentally observed peak is depicted as guide-to-the-eye curve [full width at half maximum (FWHM) $=500$ meV] (b) Local ionic coordination of bulklike (type A) and interfacial (type B) oxygen vacancies in an idealized, unrelaxed spinel/perovskite heterointerface and calculated (relative) formation enthalpy $\Delta H$ for oxygen vacancies at and away from the interface. (c) Ti $3 d$-PDOS and real-space electron density of the $\gamma-\mathrm{Al}_{2} \mathrm{O}_{3} / \mathrm{SrTiO}_{3}$ interface with one oxygen vacancy in the $\mathrm{TiO}_{2}$ layer. The real-space charge density is shown for the interfacial $\mathrm{TiO}_{2}$ layer in direct proximity to the spinel film and obtained through energy integration of the resulting IGB $\mathrm{I}_{1}$ and $\mathrm{IGB}_{2}$ state, respectively.

comes insulating when exposed to oxygen at high temperatures [3], since its interfacial 2DES essentially stems from oxygen vacancies and lacks an intrinsic component [49] despite a possible polar discontinuity [6,50]. Therefore, a different strategy is needed to achieve high mobilities in $\gamma-\mathrm{Al}_{2} \mathrm{O}_{3} / \mathrm{SrTiO}_{3}$.

We argue that, as depicted in Fig. 4(a), the oxygen vacancy concentration and distribution in low-mobility $\gamma-\mathrm{Al}_{2} \mathrm{O}_{3} / \mathrm{SrTiO}_{3}$ heterostructures is similar to that in standard $\mathrm{LaAlO}_{3} / \mathrm{SrTiO}_{3}$ samples with comparable mobilities $(\mu \approx$ $\left.1000 \mathrm{~cm}^{2} / \mathrm{V} \mathrm{s}\right)[3,48]$. In contrast, as shown in Fig. 4(b), we propose a different oxygen vacancy distribution in high-mobility $\gamma-\mathrm{Al}_{2} \mathrm{O}_{3} / \mathrm{SrTiO}_{3}$ heterostructures, where the majority of oxygen vacancies resides at the very interface (type B) and effectively acts as a single layer of electron donors. They provide the itinerant electrons that form the spatially much more extended 2DES. Experimental estimates for its depth from the interface range from 15 [6] to $75 \AA$ [51], where the amount of bulklike (type A) oxygen defects is strongly suppressed. Reminiscent of modulation-doped semiconductor structures, this spatial separation of ionized donor scattering sites and the 2DES results in a significant mobility enhancement and hence reconciles the coexistence of high mobilities with the abundance of oxygen vacancies. Note that an additional contribution to the mobility enhancement may be the increased electronic screening of electron-phonon interactions, that suppresses the formation of polarons with an enhanced effective mass in $\mathrm{SrTiO}_{3}$-based structures [52-54].

Our model also offers a natural explanation for the extremely narrow parameter window for the formation of a highmobility 2DES [4]. Pulsed laser deposition (PLD) of $\gamma-\mathrm{Al}_{2} \mathrm{O}_{3}$ thin films on $\mathrm{SrTiO}_{3}$ results in an oxygen vacancy distribution, which is determined by the intricate interplay between bulk and interface oxygen vacancy formation during the deposition process and by their redistribution during cooldown. Naturally, small deviations from the optimum growth conditions (e.g., oxygen pressure, temperature, and laser fluency) will result in (a)

$\mathrm{LaAlO}_{3} / \mathrm{SrTiO}_{3}$

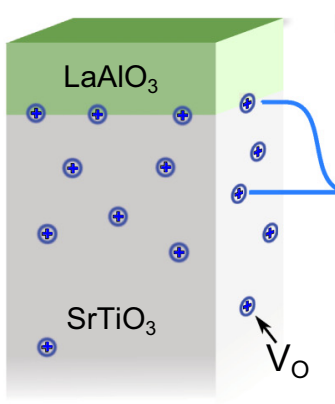

Low- $\mu$ - $-\mathrm{Al}_{2} \mathrm{O}_{3} / \mathrm{SrTiO}_{3}$

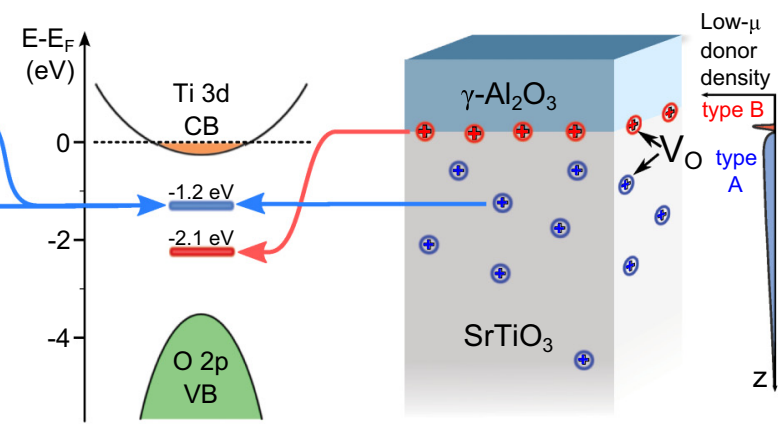

(b)

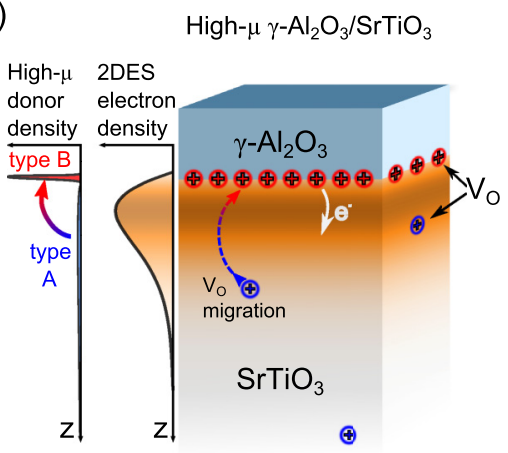

FIG. 4. (a) Schematic comparison of oxygen vacancy distribution and energetics in $\mathrm{LaAlO}_{3} / \mathrm{SrTiO}_{3}$ and low-mobility $\gamma$ - $\mathrm{Al}_{2} \mathrm{O}_{3} / \mathrm{SrTiO}_{3}$. Possible intrinsic doping mechanisms associated with the polar discontinuity have been omitted here. (b) Proposed mechanism for high-mobility conductivity in $\gamma-\mathrm{Al}_{2} \mathrm{O}_{3} / \mathrm{SrTiO}_{3}$ heterostructures. The interfacial oxygen vacancies act as the dopant layer, which is spatially separated from the 2DES in the deeper-lying layers of $\mathrm{SrTiO}_{3}$, where ionized donor scattering becomes minimized. Gradual oxygen vacancy diffusion towards the interface during annealing or room-temperature storage changes the oxygen vacancy density from the depicted low- $\mu$ into a high- $\mu$ density distribution. 
oxygen vacancies in the $2 \mathrm{DES}$ region, enhanced ionized donor scattering, and a lower mobility. Likewise, the postgrowth mobility enhancement during room-temperature storage can be explained by a gradual oxygen vacancy migration towards the energetically favorable interface, as depicted in Fig. 4(b), resulting in a reduced scattering of the 2DES electrons while conserving the total amount of donors.

Oxygen vacancies at the $\mathrm{LaAlO}_{3} / \mathrm{SrTiO}_{3}$ heterointerface have been associated with ferromagnetism, i.e., ordered local magnetic moments trapped at neighboring $\mathrm{Ti}$ sites $[55,56]$. We speculate that oxygen vacancies at the spinelperovskite $\gamma-\mathrm{Al}_{2} \mathrm{O}_{3} / \mathrm{SrTiO}_{3}$ interface may also favor magnetic ordering that could qualitatively differ from their deeper-lying counterparts and $\mathrm{LaAlO}_{3} / \mathrm{SrTiO}_{3}$. A careful experimental investigation of the magnetic properties offers a promising path to a more complete insight into the physics of this intriguing system.

This work was supported by the Deutsche Forschungsgemeinschaft (SFB 1170, SFB/TRR 49, FOR 1162, and FOR 1346) and the German Federal Ministry for Education and Research (05K13WW1). We thank G. Sangiovanni for fruitful discussions and D. McCue for his technical support during the DLS I09 beamtimes.
[1] J. Mannhart and D. G. Schlom, Science 327, 1607 (2010).

[2] S. Thiel, G. Hammerl, A. Schmehl, C. W. Schneider, and J. Mannhart, Science 313, 1942 (2006).

[3] Y. Z. Chen, N. Bovet, F. Trier, D. V. Christensen, F. M. Qu, N. H. Andersen, T. Kasama, W. Zhang, R. Giraud, J. Dufouleur, T. S. Jespersen, J. R. Sun, A. Smith, J. Nygård, L. Lu, B. Büchner, B. G. Shen, S. Linderoth, and N. Pryds, Nat. Commun. 4, 1371 (2013).

[4] Y. Z. Chen, N. Bovet, T. Kasama, W. W. Gao, S. Yazdi, C. Ma, N. Pryds, and S. Linderoth, Adv. Mater. 26, 1462 (2013).

[5] N. Nakagawa, H. Y. Hwang, and D. A. Muller, Nat. Mater. 5, 204 (2006).

[6] P. Schütz, F. Pfaff, P. Scheiderer, Y. Z. Chen, N. Pryds, M. Gorgoi, M. Sing, and R. Claessen, Phys. Rev. B 91, 165118 (2015).

[7] L. Yu and A. Zunger, Nat. Commun. 5, 5118 (2014) .

[8] P. Scheiderer, F. Pfaff, J. Gabel, M. Kamp, M. Sing, and R. Claessen, Phys. Rev. B 92, 195422 (2015).

[9] G. Herranz, F. Sánchez, N. Dix, M. Scigaj, and J. Fontcuberta, Sci. Rep. 2, 758 (2012).

[10] Z. Q. Liu, C. J. Li, W. M. Lü, X. H. Huang, Z. Huang, S. W. Zeng, X. P. Qiu, L. S. Huang, A. Annadi, J. S. Chen, J. M. D. Coey, T. Venkatesan, and Ariando, Phys. Rev. X 3, 021010 (2013).

[11] A. F. Santander-Syro, O. Copie, T. Kondo, F. Fortuna, S. Pailhès, R. Weht, X. G. Qiu, F. Bertran, A. Nicolaou, A. Taleb-Ibrahimi, P. Le Fèvre, G. Herranz, M. Bibes, N. Reyren, Y. Apertet, P. Lecoeur, A. Barthélémy, and M. J. Rozenberg, Nature (London) 469, 189 (2011).

[12] W. Meevasana, P. D. C. King, R. H. He, S.-K. Mo, M. Hashimoto, A. Tamai, P. Songsiriritthigul, F. Baumberger, and Z.-X. Shen, Nat. Mater. 10, 114 (2011).

[13] S. M. Walker, F. Y. Bruno, Z. Wang, A. de la Torre, S. Riccó, A. Tamai, T. K. Kim, M. Hoesch, M. Shi, M. S. Bahramy, P. D. C. King, and F. Baumberger, Adv. Mater. 27, 3894 (2015).

[14] V. N. Strocov, T. Schmitt, U. Flechsig, T. Schmidt, A. Imhof, Q. Chen, J. Raabe, R. Betemps, D. Zimoch, J. Krempasky, X. Wang, M. Grioni, A. Piazzalunga, and L. Patthey, J. Synchrotron Radiat. 17, 631 (2010).

[15] V. N. Strocov, X. Wang, M. Shi, M. Kobayashi, J. Krempasky, C. Hess, T. Schmitt, and L. Patthey, J. Synchrotron Radiat. 21, 32 (2013).

[16] J. P. Perdew, K. Burke, and M. Ernzerhof, Phys. Rev. Lett. 77, 3865 (1996).

[17] P. E. Blöchl, Phys. Rev. B 50, 17953 (1994).
[18] G. Kresse and D. Joubert, Phys. Rev. B 59, 1758 (1999).

[19] G. Kresse and J. Furthmüller, Phys. Rev. B 54, 11169 (1996).

[20] J. Hafner, J. Comput. Chem. 29, 2044 (2008).

[21] A. I. Liechtenstein, V. I. Anisimov, and J. Zaanen, Phys. Rev. B 52, R5467 (1995).

[22] K. Koepernik and H. Eschrig, Phys. Rev. B 59, 1743 (1999).

[23] S. Okamoto, A. J. Millis, and N. A. Spaldin, Phys. Rev. Lett. 97, 056802 (2006).

[24] L. Bellaiche and D. Vanderbilt, Phys. Rev. B 61, 7877 (2000).

[25] See Supplemental Material at http://link.aps.org/supplemental/ 10.1103/PhysRevB.96.161409 for theoretical and experimental details.

[26] F. Gunkel, S. Wicklein, S. Hoffmann-Eifert, P. Meuffels, P. Brinks, M. Huijben, G. Rijnders, R. Waser, and R. Dittmann, Nanoscale 7, 1013 (2014).

[27] A. Verma, A. P. Kajdos, T. A. Cain, S. Stemmer, and D. Jena, Phys. Rev. Lett. 112, 216601 (2014).

[28] E. Ertekin, V. Srinivasan, J. Ravichandran, P. B. Rossen, W. Siemons, A. Majumdar, R. Ramesh, and J. C. Grossman, Phys. Rev. B 85, 195460 (2012).

[29] C. Lin, C. Mitra, and A. A. Demkov, Phys. Rev. B 86, 161102 (2012).

[30] C. Lin and A. A. Demkov, Phys. Rev. Lett. 111, 217601 (2013).

[31] Y. Aiura, I. Hase, H. Bando, T. Yasue, T. Saitoh, and D. S. Dessau, Surf. Sci. 515, 61 (2002).

[32] S. McKeown Walker, A. de la Torre, F. Y. Bruno, A. Tamai, T. K. Kim, M. Hoesch, M. Shi, M. S. Bahramy, P. D. C. King, and F. Baumberger, Phys. Rev. Lett. 113, 177601 (2014).

[33] G. Berner, M. Sing, H. Fujiwara, A. Yasui, Y. Saitoh, A. Yamasaki, Y. Nishitani, A. Sekiyama, N. Pavlenko, T. Kopp, C. Richter, J. Mannhart, S. Suga, and R. Claessen, Phys. Rev. Lett. 110, 247601 (2013).

[34] G. Drera, F. Banfi, F. F. Canova, P. Borghetti, L. Sangaletti, F. Bondino, E. Magnano, J. Huijben, M. Huijben, G. Rijnders, D. H. A. Blank, H. Hilgenkamp, and A. Brinkman, Appl. Phys. Lett. 98, 052907 (2011).

[35] G. Drera, G. Salvinelli, F. Bondino, E. Magnano, M. Huijben, A. Brinkman, and L. Sangaletti, Phys. Rev. B 90, 035124 (2014).

[36] M. L. Knotek and P. J. Feibelman, Phys. Rev. Lett. 40, 964 (1978). 
[37] L. Dudy, M. Sing, P. Scheiderer, J. D. Denlinger, P. Schütz, J. Gabel, M. Buchwald, C. Schlueter, T.-L. Lee, and R. Claessen, Adv. Mater. 28, 7443 (2016) .

[38] M. Sing, G. Berner, K. Goß, A. Müller, A. Ruff, A. Wetscherek, S. Thiel, J. Mannhart, S. A. Pauli, C. W. Schneider, P. R. Willmott, M. Gorgoi, F. Schäfers, and R. Claessen, Phys. Rev. Lett. 102, 176805 (2009).

[39] S. Tanuma, C. J. Powell, and D. R. Penn, Surf. Interface Anal. 35, 268 (2003).

[40] H. O. Jeschke, J. Shen, and R. Valentí, New J. Phys. 17, 023034 (2015).

[41] R. S. Zhou and R. L. Snyder, Acta Crystallogr., Sect. B 47, 617 (1991).

[42] B. Ealet, M. H. Elyakhloufi, E. Gillet, and M. Ricci, Thin Solid Films 250, 92 (1994).

[43] S.-D. Mo, Y.-N. Xu, and W.-Y. Ching, J. Am. Ceram. Soc. 80, 1193 (1997).

[44] Y. Cao, X. Liu, P. Shafer, S. Middey, D. Meyers, M. Kareev, Z. Zhong, J.-W. Kim, P. J. Ryan, E. Arenholz, and J. Chakhalian, npj Quantum Mater. 1, 16009 (2016).

[45] D. V. Christensen, M. von Soosten, F. Trier, T. S. Jespersen, A. Smith, Y. Chen, and N. Pryds, Adv. Electron. Mater. 3, 1700026 (2017).

[46] M. Huijben, A. Brinkman, G. Koster, G. Rijnders, H. Hilgenkamp, and D. H. A. Blank, Adv. Mater. 21, 1665 (2009).

[47] J. Gabel, M. Zapf, P. Scheiderer, P. Schütz, L. Dudy, M. Stübinger, C. Schlueter, T.-L. Lee, M. Sing, and R. Claessen, Phys. Rev. B 95, 195109 (2017).
[48] M. Huijben, G. Koster, M. K. Kruize, S. Wenderich, J. Verbeeck, S. Bals, E. Slooten, B. Shi, H. J. A. Molegraaf, J. E. Kleibeuker, S. van Aert, J. B. Goedkoop, A. Brinkman, D. H. A. Blank, M. S. Golden, G. van Tendeloo, H. Hilgenkamp, and G. Rijnders, Adv. Funct. Mater. 23, 5240 (2013).

[49] F. Gunkel, S. Hoffmann-Eifert, R. A. Heinen, D. V. Christensen, Y. Z. Chen, N. Pryds, R. Waser, and R. Dittmann, ACS Appl. Mater. Interfaces 9, 1086 (2016).

[50] D. V. Christensen and A. Smith, Appl. Surf. Sci. 423, 887 (2017),

[51] M. Yazdi-Rizi, P. Marsik, B. P. P. Mallett, A. Dubroka, D. V. Christensen, Y. Z. Chen, N. Pryds, and C. Bernhard, Europhys. Lett. 113, 47005 (2016)

[52] Z. Wang, S. McKeown Walker, A. Tamai, Y. Wang, Z. Ristic, F. Y. Bruno, A. de la Torre, S. Riccò, N. C. Plumb, M. Shi, P. Hlawenka, J. Sánchez-Barriga, A. Varykhalov, T. K. Kim, M. Hoesch, P. D. C. King, W. Meevasana, U. Diebold, J. Mesot, B. Moritz, T. P. Devereaux, M. Radovic, and F. Baumberger, Nat. Mater. 15, 835 (2016).

[53] Y. J. Chang, A. Bostwick, Y. S. Kim, K. Horn, and E. Rotenberg, Phys. Rev. B 81, 235109 (2010).

[54] C. Cancellieri, A. S. Mishchenko, U. Aschauer, A. Filippetti, C. Faber, O. S. Barišić, V. A. Rogalev, T. Schmitt, N. Nagaosa, and V. N. Strocov, Nat. Commun. 7, 10386 (2016).

[55] B. Kalisky, J. A. Bert, B. B. Klopfer, C. Bell, H. K. Sato, M. Hosoda, Y. Hikita, H. Y. Hwang, and K. A. Moler, Nat. Commun. 3, 922 (2012).

[56] N. Pavlenko, T. Kopp, E. Y. Tsymbal, G. A. Sawatzky, and J. Mannhart, Phys. Rev. B 85, 020407 (2012). 